\title{
New classification criteria for autoinflammatory periodic fevers
}

U sing data from the Eurofever registry, provisional evidencebased clinical classification criteria for the major monogenic autoinflammatory periodic fevers have been published in Annals of the Rheumatic Diseases by the Paediatric Rheumatology International Trials Organisation (PRINTO). Unlike previous criteria, "The size of the Eurofever registry enabled common data collection and comparison of patients with different diseases and geographical and ethnic distribution," comments corresponding author Marco Gattorno.

Hal Hoffman, an independent expert on inflammatory disorders, endorses the need for new classification criteria. He says, "Only a few diagnostic criteria are available and these have not been validated in different populations. This study is novel because it is the first methodical and evidence-based approach to determine the clinical features that are the most discerning."

The Eurofever registry includes data from 2,556 patients at 91 centres in 56 countries. The researchers designated a reference 'goldstandard' group $(n=518)$ of patients with confirmed molecular diagnosis for one of four diseases: familial Mediterranean fever (FMF; $n=291$ ),

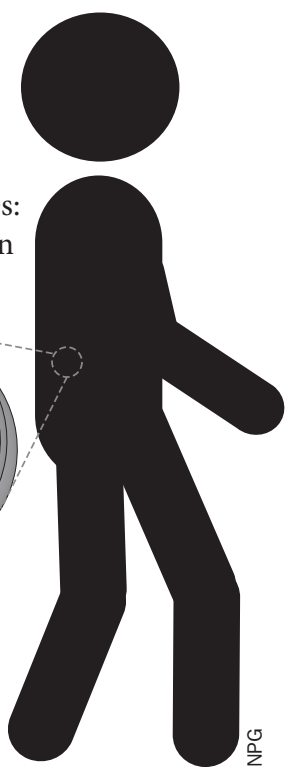

mevalonate kinase deficiency (MKD; $n=74$ ), TNF-receptor-associated periodic fever syndrome (TRAPS; $n=86$ ) or cryopyrin-associated periodic syndromes (CAPS; $n=67$ ).

Gold-standard was defined as confirmatory genetic results of two $M E F V$ mutations ( $\geq 1$ in exon 10) for FMF, two $M V K$ mutations (excluding variants with an unknown role in pathogenesis) for MKD, heterozygous TNFRSF1A mutations (excluding low-penetrance or unknown mutations) for TRAPS, and heterozygous NLRP3 mutations (excluding functional polymorphisms and variants with either low-penetrance or an unknown role in pathogenesis) for CAPS. Patients with chronic (nonperiodic) disease were excluded.

To establish the classification criteria, the researchers compared the four goldstandard groups with a control group of patients with periodic fever, apthosis, pharyngitis and adenitis (PFAPA; $n=199$ ), a non-monogenic disease. The groups were then split randomly into two sets, a training set $(n=412)$, to establish the classification criteria, and a validation set $(n=305)$.

Univariate and multivariate analysis of clinical variables between the four diseases in the training set enabled the formulation of weighted classification scores totalling 100-110 points for each disease. For example, variables included absence of chest pain (11) and presence of painful lymph nodes (13) for MKD (cut-off $\geq 42$ ), absence of vomiting (14) and presence of perorbital oedema (21) in TRAPS (cut-off $\geq 43$ ), absence of adominal pain (15) and presence of urticarial rash (25) for CAPS (cut-off $\geq 52$ ), and the absence of urticarial rash (15) and presence of abdominal pain (9) in FMF (cut-off $\geq 60$ ). All criteria had high sensitivity and specificity in the gold-standard groups.

Importantly, the criteria also performed well when applied to the group of patients who had been excluded from the testing set owing to nonconfirmatory genetic results (specificity: FMF, 87\%; TRAPS, 84\%; CAPS, 95\%; MKD, 89\%; sensitivity: FMF, 68\%; TRAPS, 59\%; CAPS, 70\%; MKD, 53\%). However, in their paper, the authors admit these data are overstated as selection from the registry probably enriched the dataset for patients already suspected of having an autoinflammatory disease. The true test of the new criteria will, therefore, be in daily practice.

"Many patients have mutations in more than one gene," says Gattorno on the difficulty of diagnosing periodic fevers, "so nonconfirmatory genetic tests can overestimate the importance of genetic variants in patients with an unclear inflammatory phenotype. This problem will be exacerbated by next-generation sequencing. Studies to correlate and validate data from molecular and clinical analysis, like ours, will be critical for classification and understanding the effect of genetic variants."

In reference to developing the criteria beyond provisional status, Gattorno adds, "We are working to establish a consensus among clinicians and geneticists to identify definitive classification criteria by combining clinical criteria and results from genetic analysis."

Hoffman's assessment of the provisional criteria is that the benefit to experts might be limited but, he says, "This scorebased approach may help physicians who are not familiar with these diagnoses." He predicts, "An approach like this could be adapted to develop a user-friendly web or computer-based diagnostic algorithm for use by patients or physicians."

Nicholas J. Bernard 